\title{
Meningitis por Streptococcus pyogenes: informe de un caso pediátrico
}

\author{
Streptococcus pyogenes meningitis: a pediatric case report
}

\author{
Dr. Raúl O. Ruvinsky ${ }^{a, b}$, Dra. Yamila Schindler ${ }^{b}$ Dra. Gabriela Urman ${ }^{b}$, Dra. Laura Cortés Lopera ${ }^{b}$, \\ Dra. Josefina Carrano ${ }^{c}$ Dra. Ana L. Paolillo y Dr. Arnoldo Grosman ${ }^{b}$
}

\begin{abstract}
RESUMEN
La meningitis por estreptococo beta hemolítico, en particular del grupo A, o Streptococcus pyogenes, es una rara localización del grupo de infecciones invasivas por este microorganismo. Se presenta con frecuencia en niños sanos, sin factores predisponentes. Representa el 0,2-1\% de todas las meningitis. Generalmente, se instala por diseminación de la infección de un foco cercano. El agregado de clindamicina mejora la eficacia del tratamiento por su acción de inhibición de la síntesis proteica, que incluye la producción de toxina. La clindamicina incrementa la acción bactericida al actuar sobre el ribosoma bacteriano. La patogenia de la enfermedad no es clara; se propuso la asociación con exotoxinas. La coinfección con el virus influenza favorecería infecciones invasivas.

Se presenta el caso de un niño de 6 años de edad previamente sano con diagnóstico de meningitis por estreptococo beta hemolítico del grupo A, localización poco frecuente en niños. Palabras clave: meningitis, Streptococcus pyogenes, infección invasiva.
\end{abstract}

\begin{abstract}
Beta hemolytic particularly of group A Streptococcus meningitis, is a rare site of the group of invasive infections caused by this microorganism. It occurs frequently in healthy children, without predisposing factors. It represents $0.2-1 \%$ of all meningitis. It is usually installed by dissemination from a nearby focus. The addition of clindamycin improves the treatment efficacy by inhibition of bacterial protein synthesis, including toxin production. The pathogenesis of the disease is not clear, the association with exotoxins was proposed. Co-infection with the influenza virus would favor invasive infections.

We present this case of a previously healthy 6-year-old boy with a diagnosis of beta hemolytic Streptococcus meningitis group A, a rare location in children.
\end{abstract}

Keywords: meningitis, Streptococcus pyogenes, invasive infection.

http:/ / dx.doi.org/10.5546/ aap.2020.e309

a. Prof. titular consulto de Pediatría, Univ. de Buenos Aires.

b. Departamento de Salud Materno Infantil, Universidad Maimónides, Ciudad Autónoma de Buenos Aires, Argentina.

c. Obra Social de los Empleados de Comercio (OSECAC), Consultorios Externos de Pediatría.

Correspondencia:

Dr. Raúl O. Ruvinsky: rauloscarruvinsky@gmail.com

Financiamiento: Ninguno.

Conflicto de intereses: Ninguno que declarar.

Recibido: 28-5-2019

Aceptado: 16-10-2019
Cómo citar: Ruvinsky RO, Schindler Y, Urman G, Cortés Lopera L, et al. Meningitis por Streptococcus pyogenes: informe de un caso pediátrico. Arch Argent Pediatr 2020;118(3):e309-e312.

\section{INTRODUCCIÓN}

El Streptococcus pyogenes o estreptococo grupo A (EGA) es un coco Gram-positivo en cadenas que produce una zona clara de hemólisis en las placas de agar sangre (hemólisis $\beta$ ). Puede encontrarse colonizando la orofaringe y como flora de la piel. Puede causar infecciones de bajo riesgo: amigdalofaringitis, impétigo, escarlatina; con menor frecuencia, erisipela, otitis media aguda (OMA), sinusitis, mastoiditis. Se relaciona con afecciones no supurativas: fiebre reumática, glomerulonefritis posestreptocócica, en franca disminución por la indicación temprana de penicilina en casos de faringitis.

La tipificación del gen emm, factor principal de virulencia presente en la proteína M del EGA, es una herramienta epidemiológica importante por su relación con presentaciones específicas de la infección. ${ }^{1}$ Se identificaron más de 200 tipos; el emm 1 sería el más relacionado con infecciones invasivas.

El EGA puede producir infecciones invasivas siempre graves; osteomielitis, neumonía, bacteriemia, sepsis; raramente, procesos con alta mortalidad, como fascitis necrotizante, shock tóxico. ${ }^{2}$ También alejadas por compromiso inmunológico, como fiebre reumática en franca disminución. La meningitis y el absceso cerebral son localizaciones extremadamente raras en la era antibiótica, sin cambios a través del tiempo, como se informó en un estudio europeo. ${ }^{3}$ La coinfección con virus, en particular influenza, debe ser considerada como importante factor de riesgo.

El Centro de Control de Enfermedades (Centers for Disease Control and Prevention, CDC) de Atlanta, EE. UU., informó, para el año 2015, 1200000 casos anuales de meningitis bacteriana (MB), la mayoría por Streptococcus pneumoniae, Neisseria meningitidis y Listeria monocytogenes. En el mismo período, ocurrieron 15440 casos 
de infecciones invasivas por EGA: celulitis (el $39 \%$ ), bacteriemia primaria (el 19,6\%), neumonía (el 14,1\%). ${ }^{4}$ El EGA representa el 0,2-1 \% de todas las meningitis. La penicilina es electiva para el tratamiento de infecciones estreptocócicas. La clindamicina aumenta la eficacia cuando se asocia al $\beta$-lactámico.

Se reporta el caso de un niño previamente sano que desarrolló meningitis por Streptococcus pyogenes con evolución clínica favorable. El propósito de esta presentación es enfatizar que este microorganismo es también una causa de meningitis en niños sin inmunocompromiso.

\section{CASO CLÍNICO}

Niño de 6 años y 8 meses de edad que consultó por un cuadro febril con otalgia, cefalea, náuseas y vómitos, de 4 días de evolución, sin antecedentes de importancia y con vacunas completas para la edad. Fue llevado al Servicio de Urgencias por la intensificación de los vómitos. Al llegar, se comprobó somnolencia, disartria y desorientación, por lo que se decidió su internación en el Servicio de Pediatría del Hospital Español.

Con signos de síndrome meníngeo, se realizó, con la tomografía axial computada (TAC) previa del sistema nervioso central (SNC) normal, una punción lumbar, que obtuvo líquido cefalorraquídeo (LCR) patológico compatible con MB: $786 / \mathrm{mm}^{3}$ de leucocitos (glóbulos blancos -GB-); neutrófilos (N): el $36 \%$; glucosa: $14 \mathrm{mg} / \mathrm{dl}$; proteínas: 0,291 g/1. El hemograma presentó leucocitosis con reactantes de fase aguda elevados: 28860 GB; N: el $85 \%$; proteína C reactiva: 18,23 mg/l; glucemia: $144 \mathrm{mg} / \mathrm{dl}$.

En el hemocultivo por dos y el cultivo del LCR, se aisló Streptococcus pyogenes sensible a penicilina. Por presentar una infección invasiva por Streptococcus pyogenes con impacto meníngeo, fue derivado a la Unidad de Terapia Intensiva Pediátrica. Se indicó un esquema antibiótico con ceftriaxona de 14 días y clindamicina de 10 días por vía intravenosa. Los hemocultivos de control fueron negativos.

\section{EVOLUCIÓN}

A las 48 horas, presentaba una mejoría de la disartria y el nivel de conciencia. Al tercer día de internación, presentó síntomas de hipertensión endocraneana. Una nueva TAC informó edema cerebral leve, por lo que se indicó dexametasona por cuatro días. El examen oftalmológico mostró diplopía y estrabismo convergente, que sugirió parálisis del VI par craneal bilateral asociada al cuadro meníngeo, por lo que se indicó oclusión alternante de los ojos. Con el diagnóstico de MB, con buena evolución y recuperado el cuadro neurológico, se otorgó el alta luego de 14 días de internación.

A las $48 \mathrm{~h}$ del alta, reingresó por presentar fiebre, cefalea y diarrea. El citoquímico del LCR, cultivos, TAC de cerebro y resonancia magnética nuclear (RMN) completa de la columna con gadolinio fueron normales. Se consideró probable un episodio de causa viral. Concurrió a controles ambulatorios con Infectología y Pediatría en forma periódica, y permaneció asintomático, sin estrabismo ni otras secuelas. La evaluación del sistema inmunitario fue normal.

\section{DISCUSIÓN}

Un estudio en Francia analizó las características de niños con meningitis en el período 2001-2012. Se detectaron 4564 casos, el $0,7 \%$ por EGA. La edad media fue de 5,6 años. Clínicamente, fueron indistinguibles de meningitis causadas por otros patógenos. ${ }^{5}$ La tasa anual de meningitis por EGA sería de 0,06/100 000 niños por año con una mortalidad del $43 \% .^{6}$

La penicilina es electiva para infecciones por EGA por penetrar bien las meninges y no haberse detectado resistencia. La ceftriaxona es efectiva para las bacterias patógenas prevalentes en meningitis de la comunidad ${ }^{7-9}$ Cuando se aísla EGA como agente causal, el agregado de clindamicina incrementa la acción bactericida al actuar sobre el ribosoma bacteriano..$^{10}$

El mecanismo de penetración del EGA al SNC es desde un foco contiguo de la vía respiratoria, como OMA, mastoiditis, faringitis; también a partir de infecciones de la piel, impétigo, varicela, escarlatina. Se presenta, con frecuencia, en niños sanos, $\sin$ factores predisponentes. ${ }^{11}$

La patogenia de la enfermedad no es clara. El EGA produce gran variedad de enzimas y toxinas, que incluyen exotoxinas pirogénicas. Las exotoxinas A,B y C estimulan los linfocitos $\mathrm{T}$ y macrófagos, y producen shock y lesión tisular, responsables de la gravedad de las infecciones invasivas. Pueden ser demostradas por reacción en cadena de polimerasas (polymerase chain reaction; $P C R$, por sus siglas en inglés).

La variedad de formas clínicas de la infección estreptocócica en individuos infectados con la misma cepa se debería a una relación compleja entre la virulencia de la bacteria, el modo de 
infección y la respuesta del huésped. En la patogenicidad del EGA, intervienen múltiples mecanismos: adhesión, invasión, acción de superantígenos con actividad tóxica. ${ }^{3}$

La proteína M ubicada en la pared celular, codificada por el gen emm, inhibe la opsonización $\mathrm{y}$ fagocitosis en ausencia del anticuerpo anti-M específico. ${ }^{12}$ Se encuentra en desarrollo una línea informática que puede predecir los tipos de $\mathrm{emm}$ predominantes, que compara datos de la secuencia del genoma completo (WGS) con el tipo de $\mathrm{emm}$ hallado por el método tradicional. Más de 200 tipos de genes emm relacionados con proteína M fueron documentados. El tipo de emm es una importante herramienta de vigilancia epidemiológica, por su relación con presentaciones específicas de la infección.

Como la meningitis por EGA es infrecuente en niños, los datos de distribución de tipos de emm son escasos. El emm 1 es el más asociado con infecciones de vías aéreas superiores e invasivas. En un estudio, emm 1 fue uno de los 3 tipos prevalentes en 170 casos de meningitis por EGA. ${ }^{13}$ Se encuentra en evaluación una vacuna 30-valente, que tendría una cobertura del $77,8 \%{ }^{14}$

En la Argentina, el Sistema Nacional de Vigilancia de la Salud informó, para 2018, a través del Boletín Epidemiológico Nacional, con datos aportados por el Laboratorio Nacional de Referencia Dr. Carlos G. Malbrán, un incremento de infecciones invasivas causadas por EGA. En la semana epidemiológica 36, se notificaron 5 casos pediátricos de enfermedad invasiva, con 2 fallecimientos: dos residentes en la Ciudad Autónoma de Buenos Aires y 3 en la provincia de Buenos Aires. A partir de la semana 37, se incorporó la vigilancia epidemiológica para infecciones invasivas por EGA; hasta la semana 40, se registraron 23 casos de infecciones invasivas, con 8 fallecimientos (el 34,8\%), que predominaron en invierno y en el comienzo de la primavera.

Para todas las edades, los serotipos prevalentes fueron M12, M1 y M3; los últimos 2, asociados a mayor gravedad, con mortalidad cercana al $30 \%$ para la forma más grave (síndrome de shock tóxico estreptocócico, SSTE). A partir de 2017, se observó un incremento del aislamiento del serotipo M3, principalmente, en la población pediátrica; no se registraron resistencias antimicrobianas. Tuvieron antecedentes 9/23: 4 de infección respiratoria aguda viral, 2 de lesiones en la piel, 2 de varicela y 1 de escarlatina. Hubo coinfecciones en 3 casos: 2 de influenza y adenovirus, 1 de metapneumovirus. ${ }^{15}$

Cuando se aísla EGA en el LCR, se requiere detectar focos iniciales de infección en el oído, la nariz, las fauces, la piel o en los focos profundos, como mastoiditis, fístula del SNC. ${ }^{6}$ Por la baja prevalencia, el curso clínico es menos conocido que en otras MB. Pueden presentarse complicaciones: sordera, retardo mental, convulsiones. El cuadro puede ocasionar secuelas neurológicas, como alteraciones visuales y problemas de conducta, que fueron descritas en el $46 \%$ de los niños afectados. ${ }^{5,6} \mathrm{El}$ seguimiento clínico debe incluir el control de la audición y del desarrollo neurológico. El EGA es muy rara causa de $\mathrm{MB}$ en niños, con mortalidad y secuelas elevadas, por lo que se considera de interés esta presentación.

\section{REFERENCIAS}

1. Pato C, Melo-Cristino J, Ramirez M, Friäes A; Portuguese Group for the study of Streptococcal infections. Streptococcus pyogenes causing skin and soft tissue infections are enriched in the recently emerged emm 89 clade 3 and are not associated with abrogation of CovRS. Front Microbiol. 2018; 9:2372.

2. Mathur P, Arora NK, Kapil A, Das BK. Streptococcus pyogenes meningitis. Indian J Pediatr. 2004; 71(5):423-6.

3. Lamagni TL, Darenberg J, Luca-Harari B, Siljander T, et al. Epidemiology of severe S. pyogenes disease in Europe. J Clin Microbiol. 2008; 46(7):2359-67.

4. Center for Disease Control Prevention. Active bacterial core surveillance report. Emerging infections program network. Group A Streptococcus. 2015. [Acceso: 17 de octubre de 2019]. Disponibleen: https: / / www.cdc.gov/abcs / reportsfindings/survreports / gas15.pdf.

5. Levy C, BidetP, BonacorsiS, BéchetS, et al. Caractéristiques des méningites à streptocoque du groupe $\mathrm{A}$ de $1 \square$ enfant. Arch Pediatr. 2014; 21(Suppl 2):S101-3.

6. De Almeida Torres RS, Fedalto LE, De Almeida Torres $\mathrm{RF}$, Steer AC, et al. Group A streptococcus meningitis in children. Pediatr Infect Dis J. 2013; 32(2):110-4.

7. Tunkel AR, Hartman BJ, Kaplan SL, Kaufman BA, et al. Practice guidelines for the management of bacterial meningitis. Clin Infect Dis. 2004; 39(9):1267-84.

8. Kaplan EL, Johnson DR, Del Rosario MC, Horn DL. Susceptibility of group A beta-hemolytic streptococci to thirteen antibiotics: examination of 301 strains isolated in the US between 1994 and 1997. Pediatr Infect Dis J. 1999; 18(12):1069-72.

9. Núñez Ramiro AG, Adell Sales A, Calderón Fernández RJ, Frasquet J, et al. Meningitis bacteriana aguda por Streptococcus pyogenes. Ann Pediatr (Barc). 2013; 78(2):135.

10. Randhawa E, Woytanowski J, Sibliss K, Sheffer I. Streptococcus pyogenes and invasive Central Nervous System infection. SAGE Open Med Case Rep. 2018; 6:2050313X18775584.

11. Armoni MV, Bereszin E, Sáfari M, Almeida F, et al. Streptococcus pyogenes meningitis in chidren: report of two cases and literature review. Braz J Infect Dis. 2007; 11(3):375-7.

12. Tuerlinckx D, Gueulette E, Loens K, Goossens H, et al. Group A streptococcal meningitis: emm. type distribution and theoretical vaccine coverage in children. Acta Clin Belg. 2016; 71(3):138-41. 
e312 / Arch Argent Pediatr 2020;118(3):e309-e312 / Presentación de casos clínicos

13. Wong CJ, Stevens DL. Serious Group A Streptococcal Infections. Med Clin North Am. 2013; 97(4):721-36.

14. Bidet $\mathrm{Ph}$, Bonacorsi S. Facteurs de pathogénicité de Streptococcus pyogenes. Arch Pediatr. 2014;21(Suppl2):s54-61.
15. Argentina. Ministerio de Salud. Vigilancia de las enfermedades invasivas por Streptococcus Pyogenes. BES. 2018; 3(113)SE 40:20. 